\title{
Contrast of Multilayed Structures in a Field Emission Gun Scanning Electron Microscope
}

\author{
Sonia Duguey, Raynald Gauvin, Paula Horny and Thierry Wright \\ Department of Mining, Metals and Materials Engineering, McGill University, Montréal, Québec, \\ Canada, H3A 2B2.
}

A good contrast in imaging is very important to characterise materials. The choice of the detector can be very significant in determining how charging, contrast and contamination will appear. A study was conducted on the Hitachi S-4700 Field Emission Gun using the Mix Detector (MD), Lower (LD) and Upper (UD) Detectors. The feature of this microscope is the new «ExB» filter, which can be used to select the mix of electrons reaching the UD. The system can be adjusted, with the voltage bias, to give images consisting of pure Secondary Electrons (SE) to pure Backscattered Electrons (BSE), and anywhere in between. This provides great flexibility in overcoming charging and in optimizing imaging contrast.

The study was carried out on a multilayered stack grown of $\mathrm{Al}_{\mathrm{x}} \mathrm{GaAs}_{1-\mathrm{x}}(\mathrm{x}=0.40$ in atomic fraction) in a GaAs matrix used as quantum wells in the microelectronics industry. The AlGaAs line thicknesses range between 20 and $300 \mathrm{~nm}$. Several photos were taken at $5 \mathrm{keV}$ using the LD, MD and UD to see their effect on contamination squares. The UD was also used at different voltage bias. Contrary to the LD, contamination squares were detected by the UD and lightly with the MD. Very good resolution was obtained with the UD compared to the LD as shown in figure [1]. Afterwards, the UD was tested at different voltage bias as seen in figure [2]. Contamination squares were detected at $-2.5 \mathrm{~V}$ where BSE and SE with energy above this voltage were collected. At $-20 \mathrm{~V}$, only strong contamination squares were seen and finally at a bias of $-150 \mathrm{~V}$, where no SE were obtained, contamination had vanished.

Using the software Adobe Photoshop 6.0, contrast was determined for different AlGaAs line thicknesses at $5 \mathrm{keV}$ with a working distance equal to $12 \mathrm{~mm}$. These results are presented in figure [3]. To reduce the statistical noise, boxes were used in order to obtain the exact intensity. Contrast was computed by the formula : \% Contrast $=(\max -\min ) * 100 /(\min +\max )$. Except for 20 $\mathrm{nm}$, a better contrast was obtained with the UD compared to the MD and the LD. Indeed, the LD behaves as a BSE detector since it collects a fraction of $\mathrm{SE}_{\mathrm{II}}$ and $\mathrm{SE}_{\mathrm{III}}$ that comes from the BSE hitting the microscope chamber. Therefore, a noise contribution can explain that less contrast was obtained.

The UD with no bias detects only SE electrons which are about $75 \% \mathrm{SE}_{\mathrm{II}}$ and $25 \% \mathrm{SE}_{\mathrm{I}}$, according to a personal communication of D.C. Joy. As the voltage bias increases, more BSE were detected and less SE. At $-150 \mathrm{~V}$, only BSE were collected, but because of the small solid angle of the UD, the BSE detected have a strong type 1 component, giving more contrast than the pure SE signal, and, as a result, contrast increases with the voltage bias. Also shown in figure [3] is the simulated contrast for BSE electrons using the CASINO program. The concept of maximum contrast for a specific width is validated from the measurements.

\section{Acknowledgements}

The Microscopy Society of America is acknowledged for financial support through a scholarship to Sonia Duguey. Professor Eric Lifshin, from the Albany Institute of Materials, is acknowledged for providing the AlGaAs / GaAs specimen. 


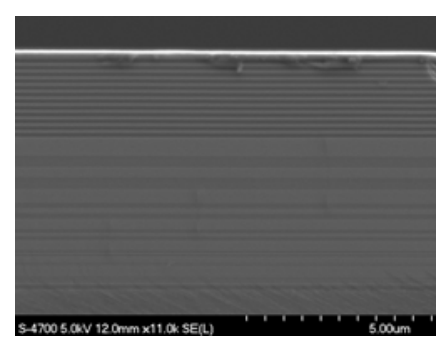

- The Lower Detector

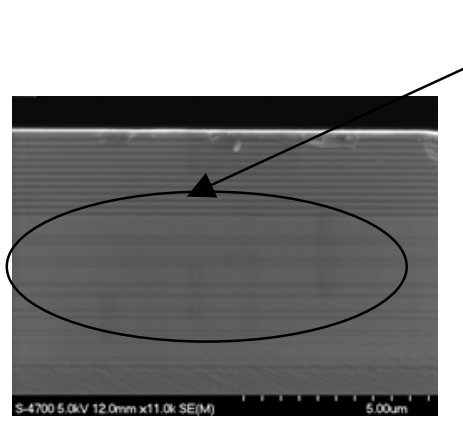

- The Mix Detector
Contamination squares

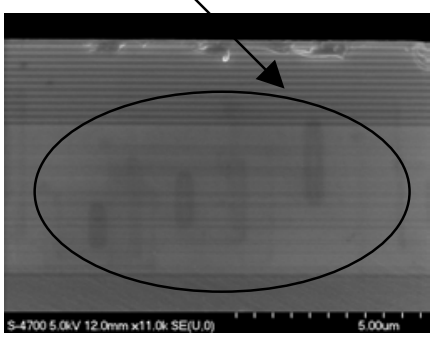

- The Upper Detector

Figure 1: Contamination effect on, in order, the lower, mix and upper detectors at $5 \mathrm{keV}-\mathrm{WD}=12 \mathrm{~mm}$.

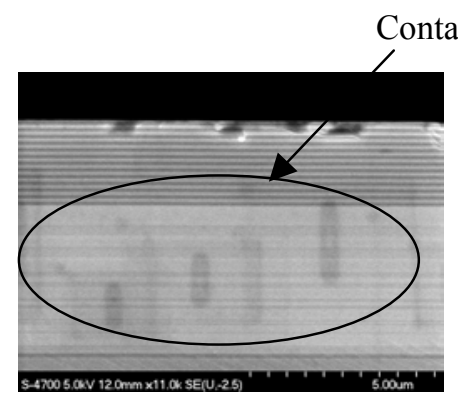

$\bullet \mathrm{UD}:-2.5 \mathrm{~V}$

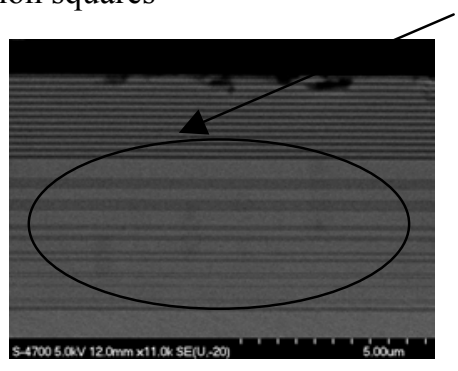

- UD : $-20 \mathrm{~V}$
Only high contamination appears

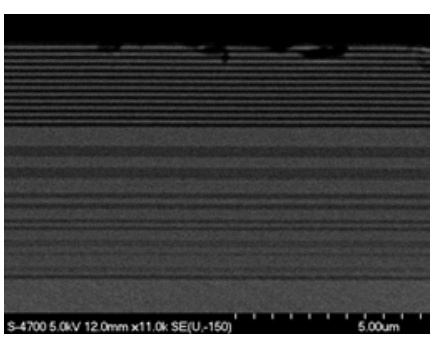

- UD : $-150 \mathrm{~V}$

Figure 2 : Contamination effect at $-2.5,-20$ and $-150 \mathrm{~V}$ on the upper detector $-5 \mathrm{keV}-\mathrm{WD}=12 \mathrm{~mm}$.

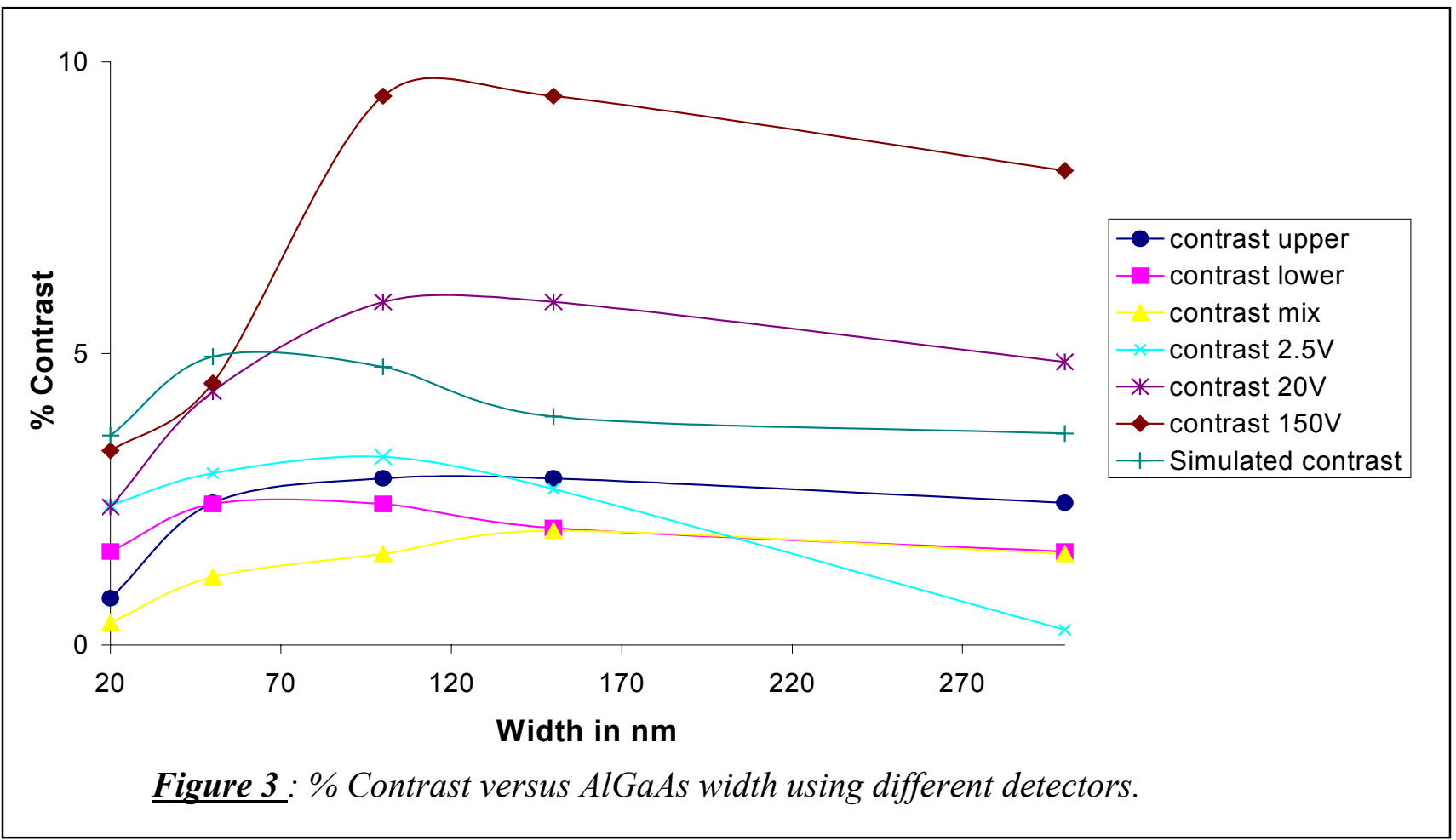

